\section{Arzneimittelprüfmodul für Gesundheitsportale}

Die ifap $\mathrm{GmbH}$ hat ein Arzneimittelprüfmodul entwickelt, das nicht nur Versandapotheken, sondern auch Gesundheitsportale als Zusatzservice für Patienten nutzen können. Bestelle ein Kunde einer Versandapotheke im Internet ein Medikament oder informiere sich darüber in einem Gesundheitsportal, prüfe das Modul „i:fox ${ }^{\circledast}$ WEB" automatisch mögliche Risiken - etwa in Bezug auf Interaktion, Kontraindikation, Schwangerschaft/Stillzeit, Doppelverordnung und Lebensmittelunverträglichkeit, so das Unternehmen. Die Rückmeldung erfolgt übersichtlich per Ampelsymbolik (www.ifap.de).

\section{Jeder Dritte Deutsche nutzt} ein Smartphone

Jeder dritte Deutsche (34\%) besitzt bereits ein Smartphone. Bei den unter 30-Jährigen soll es sogar jeder zweite sein. Das zeigt eine aktuelle repräsentative Umfrage des Marktforschungsinstituts Aris im Auftrag des Hightech-Verbands BITKOM. „Smartphones haben in wenigen Jahren den Handy-Markt aufgerollt und werden mehr und mehr zur Fernbedienung unseres Lebens", sagt BITKOM-Präsidiumsmitglied René Schuster. Das können auch Arztpraxen nutzen, indem sie ihre Praxis-Homepages mobilfähig machen oder Gesundheits-Apps in der Patientenversorgung nutzen - und sich etwa Vitaldaten per Smartphone zusenden lassen.

Software analysiert

Patientenströme im MVZ

Der Arztsoftware-Anbieter medatixx hat sein Zusatzmodul ixx.report für die ixx.concept-Editionen MVZ/Ambulanz und für ixx.vianova mit neuen Auswertungs- und Analysemöglichkeiten ausgestattet. Wie das Unternehmen meldet, könnten jetzt die ambulanten Patientenströme noch besser analysiert und optimal gelenkt werden. Das Modul zeige den Praxen nach Wunsch grafisch und tabellarisch an, zu welchen Uhrzeiten im Zeitraum einer Woche, mehrerer Wochen, eines Monats oder mehrerer Jahre wie viele Patienten ambulant in der Praxis waren. den nämlich die Bordmittel - also die Kapazitäten der TeraStation - verwendet, die die Praxis ja ohnehin für ihr Rechnernetzwerk nutzt. Zusätzliche Software musste nicht eingekauft werden. „Die Installation der Geräte war innerhalb von rund 14 Tagen abgeschlossen“, so Kuhnert.

\section{Backup-Protokoll warnt vor Datenfehlern}

Doch damit ist die Betreuung nicht am Ende: Die Ärzte haben einen LogfileSichtungsvertrag abgeschlossen. Systembetreuer Marc Kuhnert schaut sich regelmäßig - einmal pro Woche - die Protokolldateien an, die durch das Betriebssystem während der Datensicherung erstellt wurden. Er als Fachmann entschlüsselt der Praxis die teilweise kryptischen Meldungen und berichtet darüber aus- führlich an die Praxismanagerin Roswitha Faßbender. So kann das Praxisteam schnell Probleme erkennen, identifizieren und beseitigen. „Praktisch ist für uns, dass die Datensicherung vollautomatisch läuft und wir von Herrn Kuhnert wöchentlich über eine Logfile-Sichtung informiert werden“, sagt Faßbender. „Herr Kuhnert schreibt uns per Mail ein ausführliches Protokoll über die Logfiles. Das ist sehr übersichtlich und bei Problemen gut einsehbar. Besonderheiten markiert er."Um die tägliche Sicherung auf der zusätzlichen Festplatte kümmern sich die Praxismanagerin und eine Kollegin hingegen selbst. Aber auch hier läuft der Prozess weitgehend automatisiert ab, sodass durch die Datensicherung eigentlich keine Zusatzbelastung für das Praxisteam entsteht.

Thomas Jungbluth

\title{
Haben CD und DVD als Speichermedien ausgedient?
}

\author{
Jede Datensicherung ist nur so gut wie das Speichermedium. Doch worauf \\ sollten Praxisteams bei der Auswahl achten?
}

A uch in Arztpraxen nimmt die Menge der elektronischen Daten stetig zu. Aber nicht nur das hat CD-ROM und DVD den Rang als gutes Speichermedium für das Daten-Backup - also die Sicherung der Praxisdaten - abgelaufen. Noch viel schwerer wirkt folgender Nachteil der Disks: Ein automatisches Backup ist mit ihnen kaum möglich. Und damit wären wir auch schon bei einem entscheidenden Punkt für die Auswahl geeigneter BackupMedien - zumindest für große Praxiseinheiten und solche, die sich nicht ständig aktiv mit der Datensicherung beschäftigen wollen: Erlaubt das Speichermedium eine automatisierte Datensicherung, die einmal installiert wird und dann läuft, oder nicht?

Klar mit ja beantworten lässt sich die Frage für externe USB-Festplatten, die zur Datensicherung an das Praxisnetz beziehungsweise den Praxisrechner angeschlossen und beispielsweise einmal wöchentlich gewechselt werden. Denn hierauf kann durch entsprechende Sicherungsprogramme oder -befehle direkt und automatisch zugegriffen werden. Ak- tiv irgendwelche Tasten betätigen müssen die Praxismitarbeiterinnen dafür in der Regel nicht. Diese USB-Platten sind schon ab rund $60 € \mathrm{zu}$ haben, mit einer Speicherkapazität von 1 Terabyte. Noch ein Vorteil: Wie CD oder DVD können sie ohne Aufwand außerhalb der Praxis aufbewahrt werden, dazu müssen die Platten nur abgestöpselt werden. Aber: Die externen Festplatten sind relativ stoßanfällig. Fallen sie herunter, könnte ihre Funktionsfähigkeit und damit die Datensicherung leiden.

Eine interessante Alternative sind Netzwerkspeicher - kurz NAS (Network Attached Storage). Diese Geräte gibt es ebenfalls schon für Kapazitäten von 1 und 2 Terabyte, jedoch kosten sie meist $300 €$ und mehr. Da hier die Daten innerhalb des NAS noch einmal gespiegelt werden, sind sie sozusagen mehrfach geschützt.

Eine weitere Methode der Datensicherung wäre noch die Datensicherung auf Bändern, die aber den Nachteil hat, dass diese ebenfalls bereits bei leichter Beschädigung störanfällig werden.

Rebekka Höhl 